\begin{tabular}{lcr}
\hline \multicolumn{3}{c}{ ANNALES } \\
UNIVERSITATIS MARIAE CURIE-SKŁODOWSKA & \\
LOL. LXXI, 1 & LUBLIN - POLONIA & \\
\hline
\end{tabular}

\title{
Comparison of contact angle measurement methods of liquids on metal alloys
}

\author{
Konrad Terpiłowski ${ }^{\text {a, }}$, , Lucyna Hołysz ${ }^{\mathrm{a}}$, Diana Rymuszka ${ }^{\mathrm{a}}$ \\ and Robert Banach ${ }^{\mathrm{b}}$ \\ ${ }^{a}$ Department of Physical Chemistry-Interfacial Phenomena, \\ Faculty of Chemistry, Maria Curie-Sklodowska University, \\ 20-031 Lublin, Poland \\ ${ }^{b}$ Boryszew ERG Sochaczew \\ "e-mail:terpil@umcs.pl
}

The paper presents the studies of metal wettability using two methods: the sessile droplet and immersion ones. Based on the measured contact angles, there was calculated apparent surface free energy from the acidic-basic approach and the contact angle hysteresis. The advancing contact angles measured using the immersion method exhibit a little higher values than those measured by the sessile droplet method. The application of the immersion method leads to obtaining higher contact angle hysteresis. Both methods give different values of contact angles but they can be applied independently for estimation of metal surface wettability.

\section{INTRODUCTION}

The phenomenon of solid wettability by liquids is one of the commonly applied in nature and human life. Despite such great significance of wettability phenomena, their mechanisms are not fully known. Contact angle and apparent surface free energy are physical 
quantities describing wettability. In 1805 Young [1] found the dependence between the contact angle and apparent surface free energy of the solid. There are numerous theoretical approaches enabling estimation of surface free energy [2-6]. However, none of them does not provide final solution. The way of contact angle measurements in the literature is a controversial subject $[7,8]$. In spite of the problems connected with estimation of the apparent surface free energy, it is one of the most frequently determined quantity in industry is usually called surface tension of solids and is estimated based on the studies of spreading of calibrated probe liquids over solids. A trial of probe liquid of known surface tension is made on the surface and if the liquid does not spread completely or converge into a droplet for a few seconds, the apparent surface free energy of a given solid is equal to the surface tension of the probe liquid. The results of such studies are insufficient and the lifetime of testers is short. This is of particular importance in printing industry where surfaces are tested, among others, before printing and the difference of apparent surface free energy value reaching a few $\mathrm{mJ} / \mathrm{m}^{2}$ can have an essential effect on paint adhesion or while protecting metals parts against corrosion. However, tracking changes of apparent surface free energy of different kinds of material subjected e.g. to etching or activation with plasma is significant importance. Apparent surface free energy is usually determined from the contact angles measurements. Contact angle is measured using special devices with a sensitive camera and suitable computer program enabling picture treatment. These devices are very expensive and often measure only contact angles. Their purchase is in most cases economically unjustified. Another method used for measuring contact angle is the immersion method using tensiometers. Compared with the apparatus used for measuring contact angle, the tensiometer is much cheaper and more universal. Besides measuring contact angles, it can be used to measure surface tension of liquid, interfacial tension, critical micellization concentration of surface active agents. Tensiometers are used more often in industry plants.

The Department of Physical Chemistry-Interfacial Phenomena, Maria Curie-Skłodowska University in Lublin has been cooperating with the industrial plants: GT85, Idea Ekotechnologie, Boryszew S.A. for many years. Measurements of both contact angles and apparent surface free energy are very important for them. The Department of Interfacial Phenomena possesses the apparatus for contact angle measurements and tensiometers. Therefore the aim of this paper was to compare the methods of contact angle measurement using the apparatus for contact angle 
measurement, GBX France, with the immersion method employing the tensiometer KSV Sigma 700. The wettability studies were carried out for the following metal plates: aluminium, copper, steel. Water, formamide, glycerol and diiodomethane were applied as test liquids.

In the two-phase system e.g. solution-gas, there are three regions whose physical properties are different. These are homogeneity of the liquid and gas phases as well as interface region whose composition differs significantly from both volumetric phase and changes with its thickness. While considering surface tension Young [1] assumed that the surface region can be treated as a membrane of finite thickness [9-11]. Energy excess of the molecules at the surface is called surface/or interface energy. The general phase energy can be presented as the sum of energy of molecules inside the phase and that of the molecules at the surface. Surface free energy is a thermodynamic function well describing the surface. Analogously to the number of moles, the free energy of the boundary surface $\mathrm{F}^{\mathrm{S}}$ can be as follows [9-12]:

$$
F^{S}=F-F^{V}-F^{L}
$$

or

$$
F^{S}=U^{S}-T S^{S}
$$

where: $F, F^{V}, F^{L}$ are the total free energy, free energy of particles in the gas and liquid phases, $U=Q+W-$ the total energy of the system (sum of heat and work), $T$ - the temperature, $S-$ the entropy. Index "s" refers to the surface.

As mentioned earlier, surface free energy is estimated based on few theoretical approaches. Van Oss, Good and Chaudhury [2, 13-15] studied the acid-base interactions (according to the Lewis theory) which are often a result of hydrogen bond presence. They expressed the surface tension as the sum of the two components: apolar Lifshitz-van der Waals $\gamma_{s}^{L W}$ and Lewis acid-base $\gamma_{i}^{A B}$.

$$
\gamma_{i}=\gamma_{i}^{L W}+\gamma_{i}^{A B}
$$

where: $\gamma_{s}^{L W}$ - the component of Lifshitz-van der Waals interactions, $\gamma_{i}^{A B}$ - the component of acid-base interactions.

Besides the dispersive interaction the component $\gamma_{s}^{L W}$ includes the dipole-dipole and induction dipole-dipole interactions which were considered earlier as the polar ones [16]. It is probable the for most systems the contribution of the Debye-Keesom system are insignificant. The constituent of acid-base interactions $\gamma_{i}^{A B}$ can be expressed as the 
geometric mean from the electron-donor parameters $\gamma_{i}^{-}$(basic) and the electron-acceptor $\gamma_{i}^{+}$(acidic):

$$
\gamma_{i}^{A B}=2\left(\gamma_{i}^{-} \gamma_{i}^{+}\right)^{1 / 2}
$$

where: $\gamma_{i}^{+}$is the electron - acceptor parameter, $\gamma_{i}^{-}-$the electron donor parameter of the acid - base interactions component $\gamma_{i}^{A B}$.

When it is assumed that the pressure of liquid film $\Pi_{e}=0$, then from the equation:

$$
(1+\cos \theta) \gamma_{L}=2\left[\left(\gamma_{S}^{L W} \gamma_{L}^{L W}\right)^{1 / 2}+\left(\gamma_{S}^{+} \gamma_{L}^{-}\right)^{1 / 2}+\left(\gamma_{S}^{-} \gamma_{L}^{+}\right)^{1 / 2}\right]
$$

and the measurements of the contact angles on the solid surface differing in liquid polarity (e.g. water, formamide, diiodomethane), it is possible to determine the Lifshitz van de Waals component as well as electron-donor and electron-acceptor parameters of apparent surface free energy solving the system of three equations.

Chibowski [5, 17-20] proposed calculation of apparent surface free energy from contact angle hysteresis, assuming that hysteresis must be dependent on apparent surface free energy. A thin layer of liquid film will be formed where are even the weakest attraction forces such as the London dispersion ones. A film will appear almost in each studied system. In such a case liquid will not interact only with the solid but the film and its pressure will play a significant role. Depending on the properties of the thin liquid layer, it can increase or decrease the value of contact angle. The above equation will assume the following form:

$$
\gamma_{S F}=\gamma_{S L}+\gamma_{L} \cos \theta_{r}
$$

but:

$$
\gamma_{S F}=\gamma_{S}+\pi
$$

where: $\gamma_{S}$ - is the apparent surface free energy resulting from the presence of the liquid film on the surface of the studied solid, $\Pi$ is the film pressure.

Thus combining the above equations, the film pressure can be calculated from following expression:

$$
\pi=\gamma_{L}\left(\cos \theta_{r}-\cos \theta_{a}\right)
$$

The using the expression for adhesion work and the Good parameter " $\Phi$ " defined by Girifalco and Good [21]. Chibowski obtained the dependence allowing to calculate the total surface free energy in the 
function of three measurable parameters: film pressure (liquid surface tension) as well as advancing and receding contact angles.

$$
\gamma_{S}^{\text {TOT }}=\gamma_{l} \frac{\left(1+\cos \theta_{a}\right)^{2}}{\left(1+\cos \theta_{r}\right)^{2}-\left(1+\cos \theta_{a}\right)^{2}}
$$

The approach proposed by Chibowski has one assumption such that the receding line of three-phase contact leaves a thin liquid layer.

\section{EXPERIMENTAL}

\subsection{Alloys plates}

To carry out the studies presented in the paper there were used the following materials:

- aluminium - the aluminium plate (0.8 mm thick), Russian production $\mathrm{Al}>93 \%$.

- steel - the plate cast using the continuous method, rolled and cut into sheet or bands. Plates and bands satisfy the requirements of the PN-EN 988 standards: The metallurgical plants Silesia S.A. of the composition: $\mathrm{Al} \approx 0.15 \%, \mathrm{Cu} \approx 0.08-1.00 \%, \mathrm{Zn} \approx 99.00 \%$ and $\mathrm{Ti} \approx 0.06-0.10 \%$.

- copper-wire rod $\mathrm{Cu}-\mathrm{ETP}-8-\mathrm{CL}$. The charge material: cathodes-sort: $\mathrm{Cu}-\mathrm{CATH}-1$ : brand: HML, HMG-B, HMG-S. They agree with standards: PN-EN 1997, ASTM-B 49, DIN-1708. Contaminants: max. 30 ppm $\mathrm{O}_{2}=170-190 \mathrm{ppm}$ (max. 400 ppm). KGHM, Polish Copper S.A. Division-Copper Steel Plant "Cedynia" in Orsk.

The plates were immersed in the wash fluid solution congaing large amounts of surfactants (Extran MA), subjected to the ultrasounds bath for 20 min. Then each plate side was washed 15 times with redistilled water purified by the Mili-Q 185 system. The washed surface were dried at $50^{\circ} \mathrm{C}(323 \mathrm{~K})$ for $40 \mathrm{~min}$. Next, the plates were taken out from drier and moved to the aseptic and tight container. They were left for $30 \mathrm{~min}$ to get the surrounding temperature. After this time (or longer) the plates were taken to measurements. Each of the studied materials was kept in a separate vessel. 


\subsection{Methods of contact angle measurements}

2.2.1. Sessile droplet method

A)

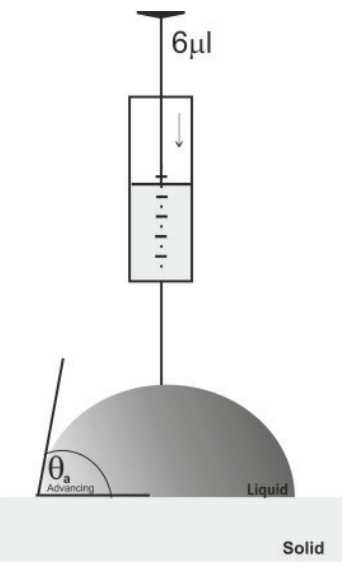

B)

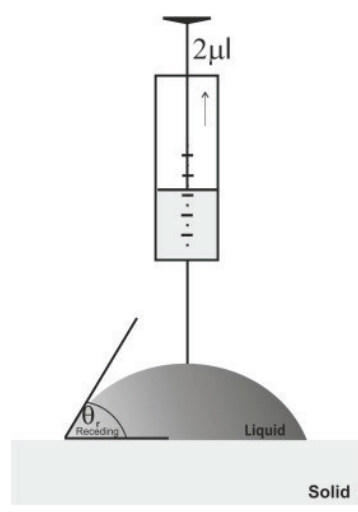

Fig. 1. Sessile droplet method of contact angle measurement.

Advancing and receding contact angles of the probe liquids on the metalsurfaces were measured using contact angle meter GBX (France) equipped with temperature and humidity controlled measuring chamber and digital camera. The measurements were conducted at $20^{\circ} \mathrm{C}$ and $50 \%$ relative humidity. A $6 \mathrm{~mm}^{3}$ [22] (Fig. 1A)) droplet from a syringe was gently settled on the sample surface with a help of an automatic deposition system.

\subsubsection{Immersion method}

A)

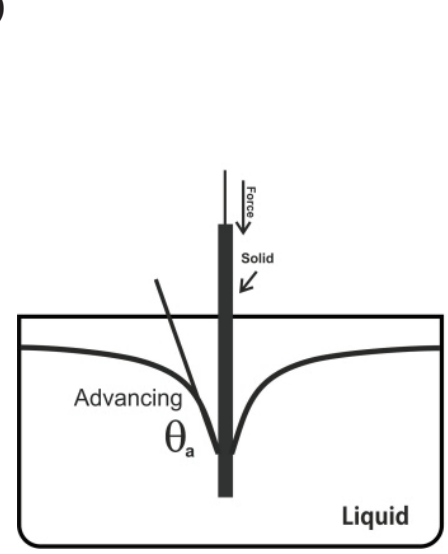

B)

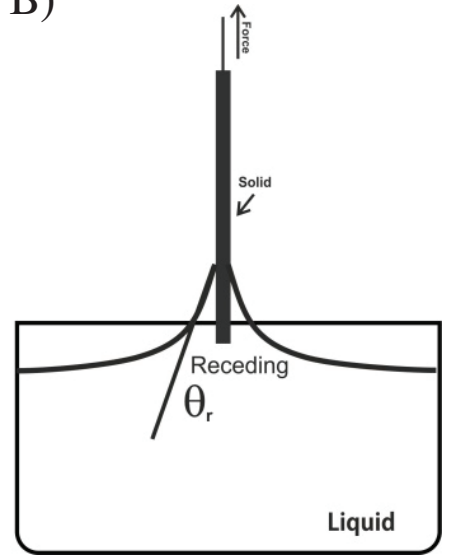

Fig. 2.Immersion method of contact angle measurement. 
The advancing contact angle was evaluated from the droplet shape by the computer program Win Drop++. Then $2 \mathrm{~mm}^{3}$ (Fig. 1B)) of the droplet volume was sucked into the syringe and the receding contact angle was calculated by the mentioned program. The advancing and receding contact angles were measured for 10 droplets of each probe liquids.

Measurements of contact angles using the immersion method were conducted applying a Tensiometer KSV Sigma 700. Before measurements the KSV Reserch Lab Sigma 70 software should be set working. After setting the program working and choosing the research method there must be collected the information about the plate and probe liquid: name of plate, name of the measurements and the measuring chosen method. The parameters of the plate on which contact angles were measured: height and thickness should be given. These parameters were measured using the slide caliper. The last parameter chosen from the program was the liquid for which the contact angles were measured. After introducing the indispensable data, the measurements were made. The rate of plate immersion (Fig. 2A) into the probe liquid was $6.0 \mathrm{~mm} / \mathrm{min}$ but rate of emergence was $7.0 \mathrm{~mm} / \mathrm{min}$. The plate was immersed to the depth of $10 \mathrm{~mm}$ (Fig. 2B). The measurement lasted about 200 seconds which made it possible to make 20 repetitions of both advancing and receding contact angles.

It is also possible to calculate the contact angle from reading the strength at any depth of plate immersion taking into account the fact that the sizes of the probe (metal plate) and the surface tension of the liquid are known. To measure contact angles, there used the following liquids: diiodomethane (D), $\mathrm{CH}_{2} \mathrm{I}_{2}(\mathrm{M}=267.84 \mathrm{~g} / \mathrm{mol}), 99 \%$, Aldrich, Germany, formamide $(\mathrm{F}), \mathrm{CH}_{3} \mathrm{NO}(\mathrm{M}=45.04 \mathrm{~g} / \mathrm{mol}), 98 \%$, Aldrich, Germany, glycerol $(\mathrm{G}), \mathrm{C}_{3} \mathrm{H}_{5}(\mathrm{OH})_{3}(\mathrm{M}=92.08 \mathrm{~g} / \mathrm{mol})$, analytically pure, $\mathrm{POCH}$ S.A, deionized water $(\mathrm{W})$ Milli-Q $185(\mathrm{pH} \approx 6.5)$.

\subsection{Apparent surface free energy estimation}

In the case of $\mathrm{CAH}$ approach surface free energy was calculated for each pair of the contact angles and then obtained amount was averaged [20].

On the other hand, surface free energy for which determination LWAB approach was used at first average advancing contact angle was calculated for every tested liquid. Next, the standard deviation was added to average advancing contact angle what leaded to obtaining the highest possible contact angle on the surface. From the average advancing contact angle, standard deviation was also subtracted and the lowest possible on the surface contact angle was obtained. Determining surface free energy, 
to equation was put the highest, average and the lowest contact angles and next average and standard deviation was calculated.

\section{RESULTS AND DISCUSSION}
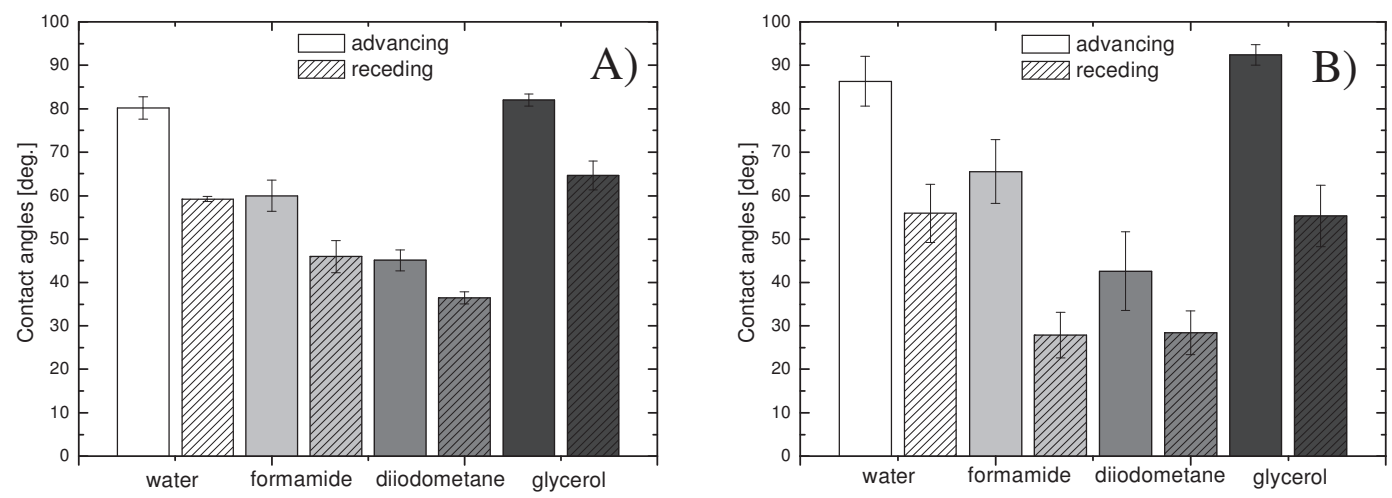

Fig. 3. Averaged results of measurements of the contact angles of: water, formamide, diiodomethane and glycerol on the steel plate by A) the method of sessile droplet B) the immersion method.
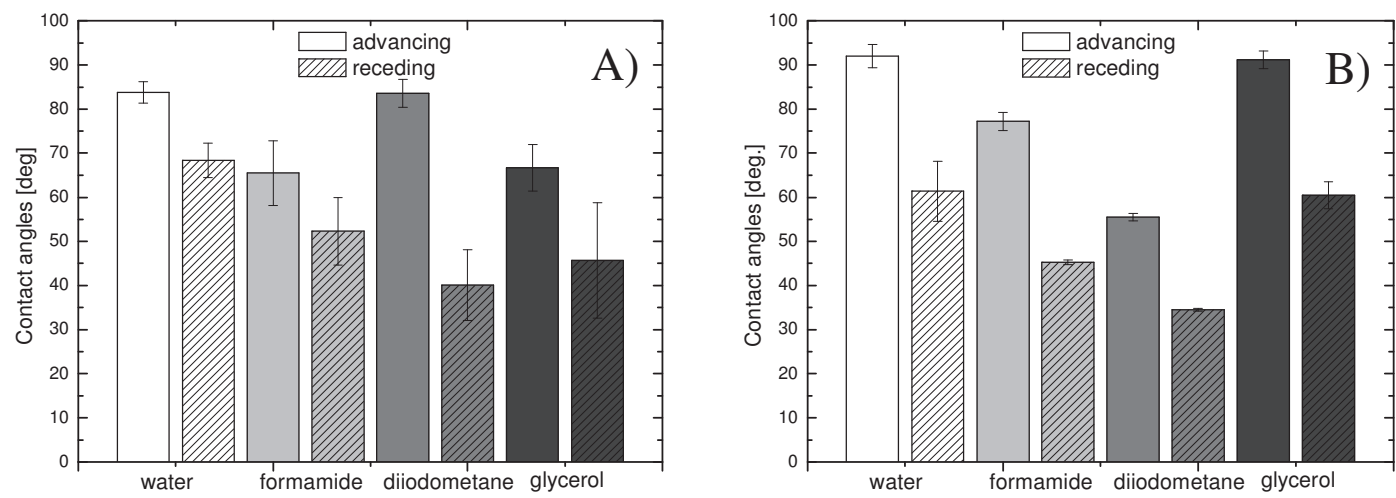

Fig. 4. Averaged results of measurements of the contact angles of: water, formamide, diiodomethane and glycerol on the cooper plate by A) the method of sessile droplet B) the immersion method.

Thought it was not possible to obtain directly comparable values of contact angles on the metallic surfaces, one was able to observe similar dependences of contact angles on all studied surfaces. The main differences between both methods consist in the fact that the immersion method gives usually higher values of advancing contact angles of all liquids which is visible in the case of the most polar liquid such as water i.e. using the sessile droplet method on steel plates and the advancing 
contact angles was $80 \pm 3.2^{\circ}$ (Fig. 4A) and 4B)) whereas using the immersion method all values all values of receding contact angles are bit lower (Figs. 3-5).
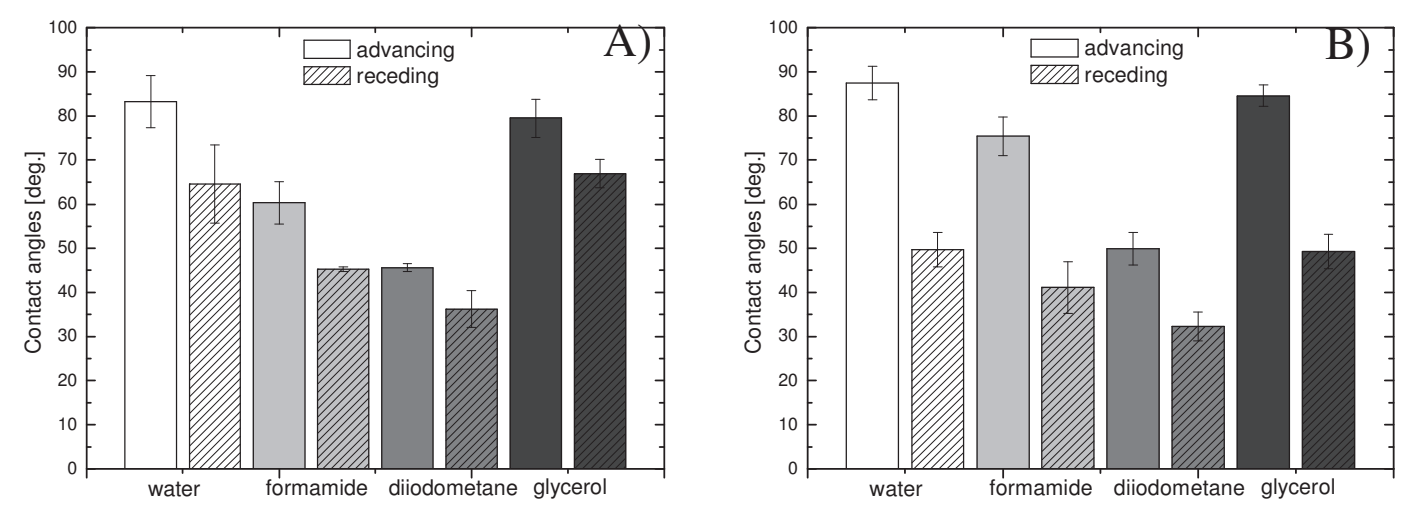

Fig. 5. Averaged results of measurements of the contact angles of: water, formamide, diiodomethane and glycerol on the aluminium plate by A) the method of sessile droplet B) the immersion method.

The only liquid for which the comparable values of advancing and receding contact angles were obtained with both methods was diiodomethane (Figs 3-5). However, it should be remembered that this liquid interact with the surface only in a dispersive way and is characterized by the lowest surface tension of all liquids studied in the paper.

When the apparent surface free energy was estimated using the LWAB approach $[2,13-15]$ and the energy was calculated independently based on the two triplets of liquids: water-formamide-diiodomethane, water-glycerol-diiodomethane, it was possible to obtain comparable values of apparent surface free energy from both triplets. Whereas considering the method of contact angle measurements and when the angles are measured by the immersion method, somewhat lower values of both total apparent surface free energy and its components are obtained. The dispersive component of apparent surface free energy (sessile droplet method) for the steel plates equals $36.6 \pm 1.3 \mathrm{~mJ} / \mathrm{m}^{2}$ (Fig. 3A) but in the case of the immersion method $38.1 \pm 3.8 \mathrm{~mJ} / \mathrm{m}^{2}$ (Fig. 3B). In most cases due to large values of contact angles the total apparent surface free energy calculated using the LWAB approach was confined to the dispersion component. Such a case is connected with the appearance of negative values of elements from the polar components which is commonly regarded as a drawback of this approach. 

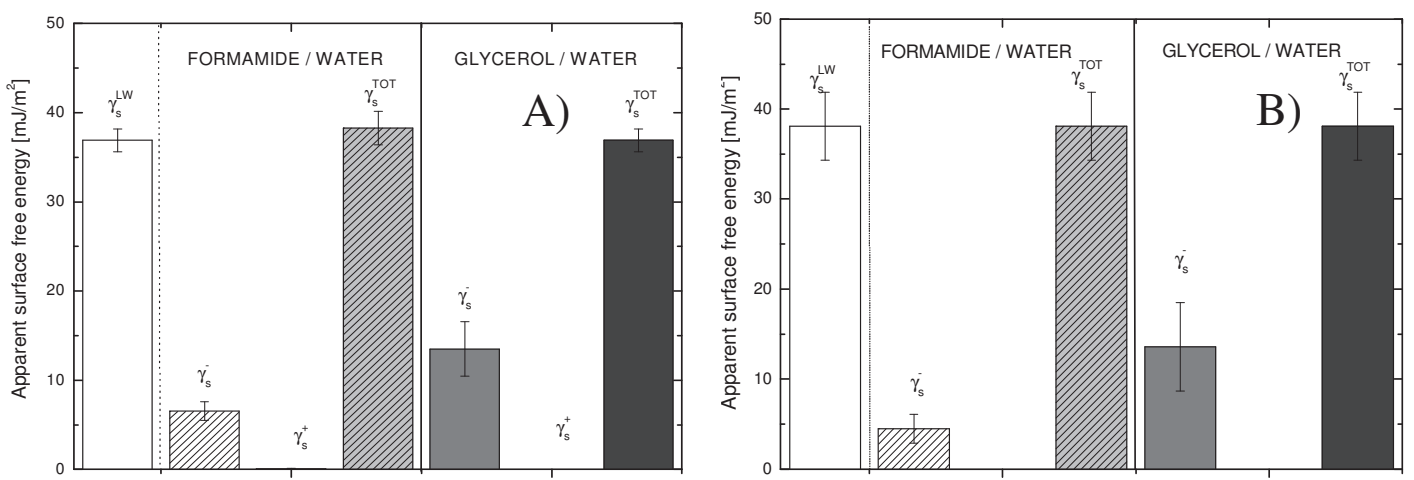

Fig. 6. Apparent surface free energy of water, formamide, diiodomethane and glycerol based on the van Oss, Good, Chaudhury equation on the surface of the steel plate by A) the sessile droplet method, B) the immersion method.
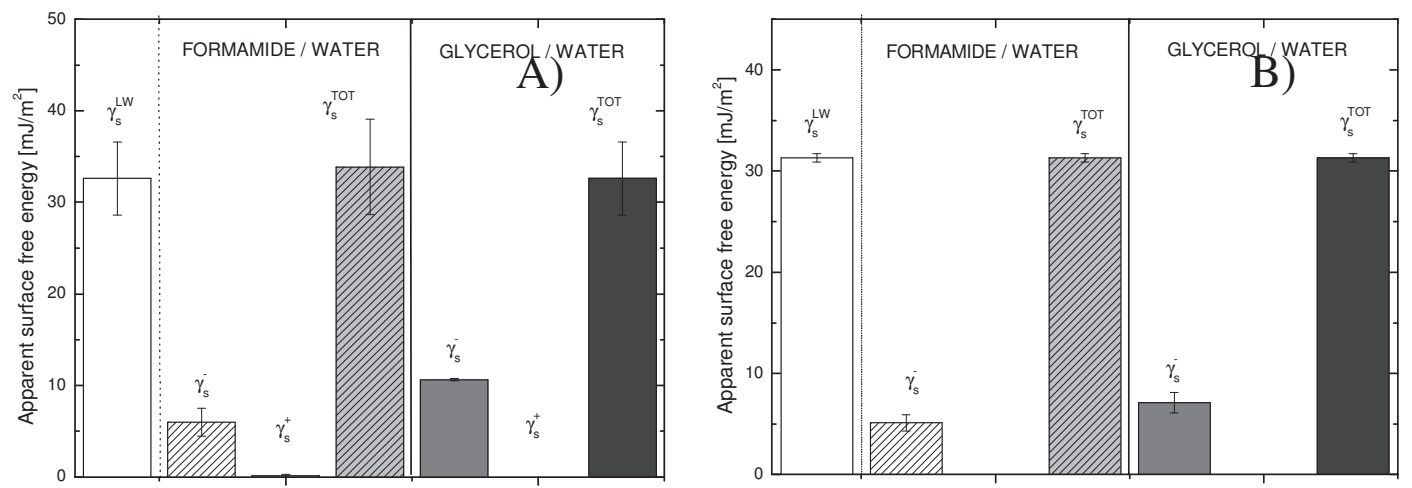

Fig. 7. Apparent surface free energy of water, formamide, diiodomethane and glycerol based on the van Oss, Good, Chaudhury equation on the surface of the copper plate by A) the sessile droplet method, B) the immersion method.
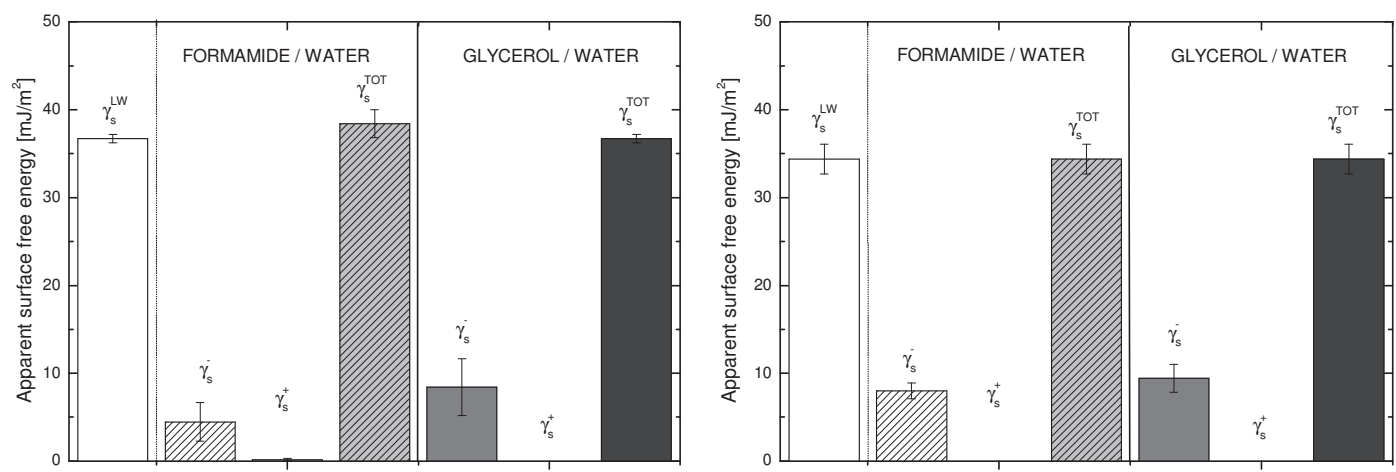

Fig. 8. Apparent surface free energy of water, formamide, diiodomethane and glycerol based on the van Oss, Good, Chaudhury equation on the surface of the aluminium plate by A) the sessile droplet method, B) the immersion method. 
As can be seen in Figs 6-8 the apparent surface free energy of metallic surfaces is composed of a dispersion component and mainly electron-donor parameter.

The problem of metal apparent surface free energy was studied by Radelczuk [23] and contact angles were measured using the sessile droplet method using other metal alloys: duraluminium D16 and steel. While measuring contact angles on steel, the advancing contact angle of water was $63.9 \pm 0.4^{\circ}$, the receding one $46.4 \pm 0.4^{\circ}$ but angle measured in the paper are: advancing $80.2 \pm 2.6^{\circ}$ and the receding one $59.2 \pm 0.6^{\circ}$. When the angles were measured by means of the immersion method, the contact angle of water was $86.3 \pm 6.8^{\circ}$ and receding one $55.9 \pm 6.7^{\circ}$ (Fig. 3). For the aluminium plates only the contact angles of nonpolar diiodomethane show convergent values. The advancing contact angle of diiodomethane was $49.0 \pm 0.7^{\circ}$ but the receding one $37.7 \pm 0.2^{\circ}$. Both in paper by Radelczuk [23] and in the discussed results, the roughness of studied materials is not taken into account but other measured values of contact angles can just come from different roughness. Analyzing the components of apparent surface free energy due to comparability of values of diiodomethane contact angles, the dispersive component is almost the same as in the quoted paper [24] that is $34.8 \mathrm{~mJ} / \mathrm{m}^{2}$ but the component for the sessile drop method is $36.9 \mathrm{~mJ} / \mathrm{m}^{2}$ (Fig. 6A) and for immersion method $34.4 \mathrm{~mJ} / \mathrm{m}^{2}$ (Fig. 6B). The electron-donor component contributes significantly to the total apparent surface free energy and is $20.2 \mathrm{~mJ} / \mathrm{m}^{2}$ (D-W-F) and $30.2 \mathrm{~mJ} / \mathrm{m}^{2}$ (D-W-G) but for studied aluminium plates using sessile droplet method, it is $6.0 \mathrm{~mJ} / \mathrm{m}^{2}$ and $10.6 \mathrm{~mJ} / \mathrm{m}^{2}$, respectively. In case of immersion method it is $8.0 \mathrm{~mJ} / \mathrm{m}^{2}$ (D-W-F) and $9.4 \mathrm{~mJ} / \mathrm{m}^{2}$ (D-W-G). Metals wettability was also studied by Kubiak et al. [24]. Contact angles were measured for $0.5 \mu 1$ droplets on the metals in the form of $10 \times 10 \times 10 \mathrm{~mm}$ cubes. The aim of the paper was to examine the effect of surface polishing i.e. decrease of roughness on wettability. The results of the studies presented in the paper should be compared with the first process described by Kubiak et al. [24]. These were untreated materials. On unmodified aluminium the advancing contact angle of water was $78.9^{\circ}$ but on the steel plates it was $93.7^{\circ}$ and on the copper $83.5^{\circ}$. Analyzing the results obtained by them, one can be seen that the roughness and even its structure have great effect on metal wettability.

When the apparent surface free energy was estimated based on the Chibowski approach [5, 13-15], the energy calculated from the contact angles measured by the immersion method had the value lower than those 
calculated from the angles measured by the sessile droplet method (Figs. 9-11). This refers mainly to the polar liquids used for measuring the contact angles. In the case when the energy is calculated as the average from energy estimated from water- diiodomethane-formamide as well as water-diiodomethane-glycerol there is achieved a good convergence of contact angle measurement methods e.g. for copper (Fig. 10) when contact angles were measured by the sessile droplet method and the averaged energy calculated from $\mathrm{CAH}$ for (W-F-D) was 35.4 $\pm 2.6 \mathrm{~mJ} / \mathrm{m}^{2}$ but for the other it was $32.7 \pm 2.8 \mathrm{~mJ} / \mathrm{m}^{2}$.

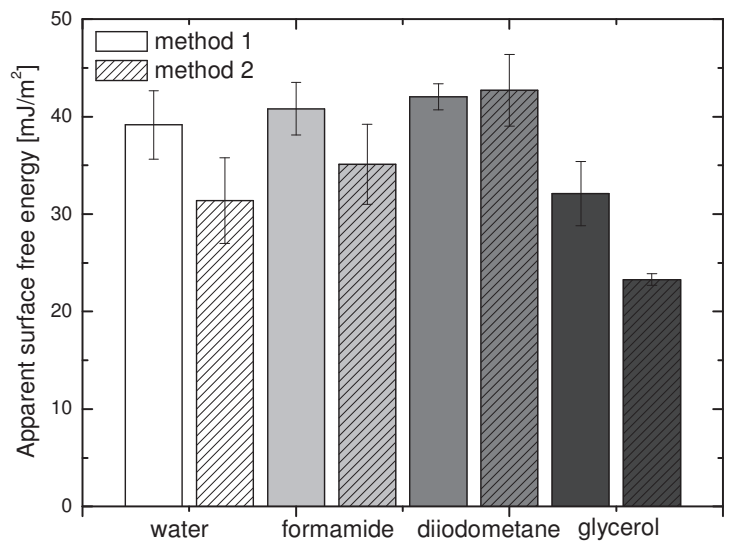

Fig. 9. Apparent surface free energy determined using Chibowski approach $(\mathrm{CAH})$. Comparison of the sessile drop method with the tensiometric one on the steel plate (method 1 - sessile droplet, method 2 -immersion one).

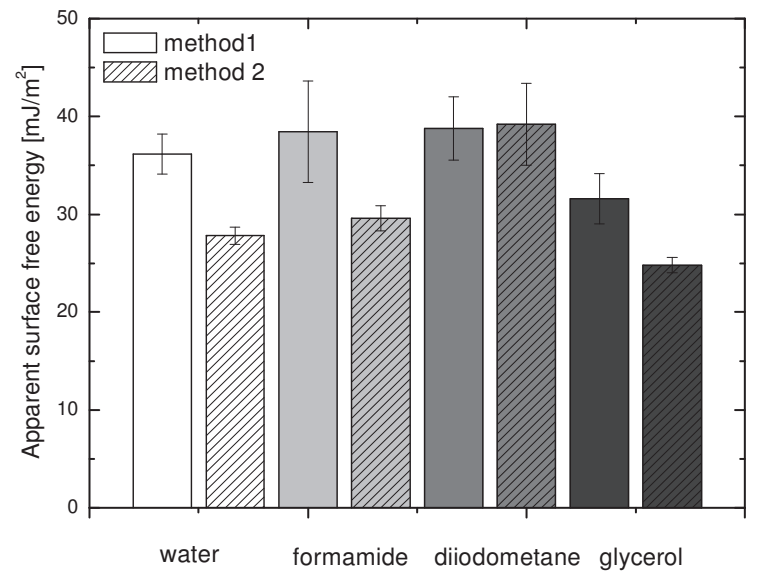

Fig. 10. Apparent surface free energy determined using Chibowski approach (CAH). Comparison of the sessile drop method with the tensiometric one on the cooper plate. 


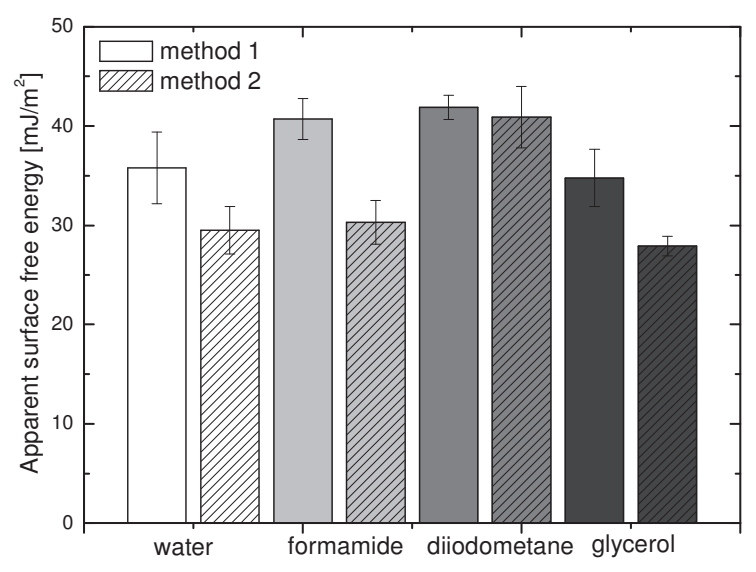

Fig. 11. Apparent surface free energy determined using Chibowski approach $(\mathrm{CAH})$. Comparison of the sessile drop method with the tensiometric one on the aluminium plate.

However, in the case of immersion method the surface free energy was $27.4 \pm 1.0 \mathrm{~mJ} / \mathrm{m}^{2}$ and $30.6 \pm 2.0 \mathrm{~mJ} / \mathrm{m}^{2}$, respectively. Generally, the smallest value are obtained for triplet: water-formamide -diiodomethane when the contact angles were measured using the immersion method. Comparing the values of calculated surface free energy obtained from the LWAB approach with those from $\mathrm{CAH}$ approach, it should be stated that the CAH approach gives larger values of apparent surface free energy to making use of both advancing and receding contact angles.

\section{CONCLUSIONS}

Contact angles of individual liquids are affected by their surface tension and components. This is evident independent of the method used for contact angle measurements. Only in case of glycerol, it has essential effect on liquid viscosity. Taking into account the obtained results it is hard to compare directly both methods. The sessile droplet method is more frequently applied in the literature but the immersion one can be applied in industry, particularly where tracking of change obtained e.g. due to surface modification is needed. Yet it should be remembered that in the immersion method both sides of the studied material must be the same. This condition largely restrict applicability of this method. Moreover, due to longer time of plate stay in the liquid whose contact angle is measured, lower values of receding contact angles are obtained because of liquid vapour adsorption on the surface. 


\section{ACKNOWLEDGEMENTS}

The eco-friendly liquid for the automotive sector with innovative environmental parameters. Chemical Advisory and Trade sp. z o.o., POIR.01.01.01-00-1738/15

\section{REFERENCES}

[1] T. Young Philos. Trans. R. Soc., London, 95, 65, (1895).

[2] C. J. van Oss, R.J. Good, M.K. Chaudhury, Langmuir, 4, 884, (1998).

[3] A.W. Neumann, R. J. Good, C. J. Hope, M. Sejpol, J. Colloid Interface Sci. 49, 291, (1974).

[4] D. K. Owens, R. C. Wendt, J. Appl. Polymer. Sci., 13, 1741, (1969).

[5] E. Chibowski, in Contact Angle, Wettability and Adhesion, K. L. Mittal, (Ed.), VSP, 2, 2, Utrecht (2002).

[6] M. Żenkiewicz, Polimery, 52(10), 760, (2007).

[7] A. Marmur, Colloid and Surfaces, 116, 55, (1996).

[8] X. Noblin, A. Buguin, F. Brochard-Wyart, Eur. Phys.J. E 14, 395, (2004).

[9] B. Jańczuk, A. Zdziennicka, W. Wójcik, Wiadomości chemiczne, 49, 5, (1995).

[10] A. W. Adamson, Physical Chemistry of Surface, Wiley-Interscience, New York, $5^{\text {th }}$ Ed., (1991).

[11] T. Young, Miscellaneous Works, G. Peacock, (Ed.), Murray, London, 1 (1855) 418.

[12] J.W. Gibbs, Collected Works, Yale University Press, New Hawen, Vol. 1, (1948) 219.

[13] C. J. van Oss, R. J. Good, M. K. Chaudhury, J. Coll. Interf. Sci. 111, 378, (1986).

[14] C. J. van Oss, R. J. Good, M. K. Chaudhury, Chem. Rev., 88, 927, (1988).

[15] R. J. Good, M. K. Chaudhury, C. J van Oss, Fundamentals of Adhesion, L. H Lee (Ed.), Plenum Press, New York (1991) pp. 153-172.

[16] O. Dredgier, A.W. Neuman, P.J. Sell, Kolloid-Z.Z. Polym. 201, 52, (1965).

[17] E. Chibowski, Adv. Colloid Interf. Sci., 133, 51, (2007).

[18] E. Chibowski, Adv. Colloid Interf. Sci., 13, 121, (2005). 
[19] E. Chibowski, Adv. Colloid Interf. Sci., 103, 149, (2003).

[20] E. Chibowski, K. Terpiłowski, Contact Angle, Wettability and Adhesion, 6, 283, (2009).

[21] Girifalco L. A., Good R. J., J. Phys. Chem., 61, 904, (1957).

[22] D. Rymuszka, K. Terpiłowski, L. Hołysz, Annales UMCS Sectio AA, 121, (2013).

[23] H. Radelczuk, L. Hołysz, E. Chibowski, J. AdhesionSci. Technol. 16, 1547, (2002).

[24] K. J. Kubiak, M. C. T. Wilson, T. G. Mathia, Ph. Carval, Wear, 271, 523, (2011).

\section{CURRICULA VITAE}

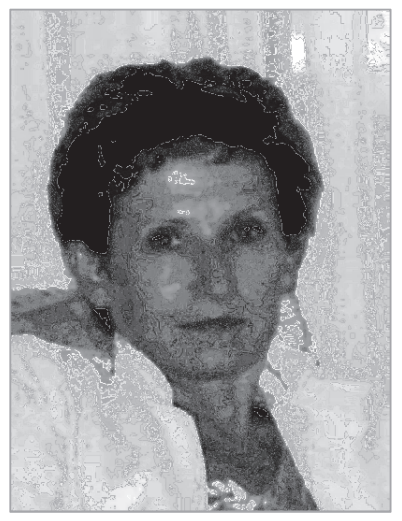

Lucyna Hotysz is a full professor and a university teacher of Physical Chemistry in the Department of Physical Chemistry-Interfacial Phenomena, Faculty of Chemistry UMCS. Her research interests is largely concentrated on interfacial phenomena and physicochemistry of the surface; physico-chemical properties of dispersed systems and their stability; the adsorption layers and the electrochemical and electrokinetic properties of solid-liquid interface; the structure and properties of the lipid layers; the preparation and characterization of superhydrophobic surfaces. She is an author and co-author of over 115 original papers in professional journals and 130 published as conference materials.

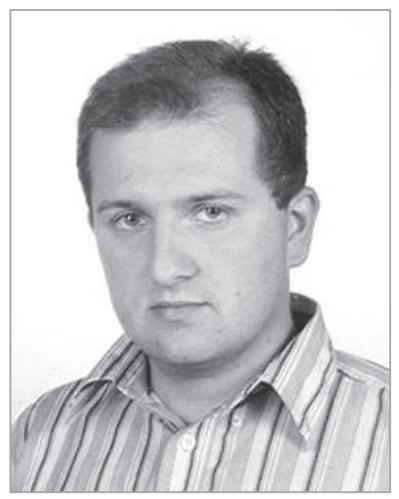

Konrad Terpitowski was born in Poland in 1979. He studied chemistry at Maria Curie Skłodowska University in Lublin and graduated in 2003. At present he is an assistant professor in the Department of Interfacial Phenomena UMCS Lublin. His research work is apparent surface free energy of solids and stability of dispersed systems. 


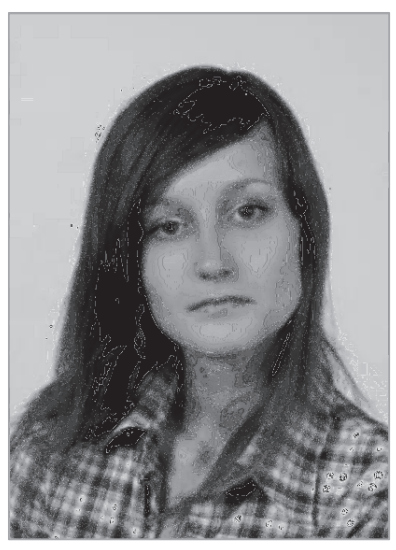

Diana Rymuszka. PhD Student of Maria CurieSkłodowska University in Lublin, Poland, Department of Interfacial Phenomena, Faculty of Chemistry.

Robert Banach Head of Research and Development in Boryszew ERG. His achievements can boast of many industrial implementations. 